\title{
Ancient, globally distributed lineage of Sarcocystis from sporocysts of the Eastern rat snake (Pantherophis alleghaniensis) and its relation to neurological sequalae in intermediate hosts
}

\author{
Shiv K. Verma ${ }^{1} \cdot$ David S. Lindsay $^{2} \cdot$ Benjamin M. Rosenthal $^{1} \cdot$ Jitender P. Dubey $^{1}$
}

Received: 2 March 2016 / Accepted: 14 March 2016/Published online: 30 April 2016

(C) Springer-Verlag Berlin Heidelberg (outside the USA) 2016

\begin{abstract}
There is an emerging concern that snakes are definitive hosts of certain species of Sarcocystis that cause muscular sarcocystosis in human and non-human primates. Other species of Sarcocystis are known to cycle among snakes and rodents, but have been poorly characterized in the USA and elsewhere. Although neurological sequalae are known for certain species of Sarcocystis, no such neurological symptoms are known to typify parasites that naturally cycle in rodents. Here, sporocysts of a species of Sarcocystis were found in the intestinal contents of a rat snake (Pantherophis alleghaniensis) from Maryland, USA. The sporocysts were orally infective for interferon gamma gene knockout (KO) mice, but not to Swiss Webster outbred mice. The KO mice developed neurological signs, and were necropsied between 33 and 52 days post-inoculation. Only schizonts/merozoites were found, and they were confined to the brain. The predominant lesion was meningoencephalitis characterized by perivascular cuffs, granulomas, and necrosis of the neuropil. The schizonts and merozoites were located in neuropil, and apparently extravascular. Brain homogenates from infected $\mathrm{KO}$ mice were infective to $\mathrm{KO}$ mice and CV-1 cell line. DNA extracted from the infected mouse brain, and infected cell cultures revealed the highest identity with Sarcocystis species that employ snakes as definitive hosts. This is the first report of Sarcocystis infection in the endangered
\end{abstract}

Jitender P. Dubey

Jitender.Dubey@ars.usda.gov

1 United States Department of Agriculture, Agricultural Research Service, Beltsville Agricultural Research Center, Animal Parasitic Diseases Laboratory, Building 1001, Beltsville, MD 20705-2350, USA

2 Department of Biomedical Science and Pathology, VirginiaMaryland Regional College of Veterinary Medicine, Virginia Tech, 1410 Prices Fork Road, Blacksburg, VA 24061, USA rat snake (P. alleghaniensis) and the first report of neurological sarcocystosis in mice induced by feeding sporocysts from a snake. These data underscore the likelihood that parasites in this genus that employ snakes as their definitive hosts constitute an ancient, globally distributed monophyletic group. These data also raise the possibility that neurological sequalae may be more common in intermediate hosts of Sarcocystis spp. than has previously been appreciated.

Keywords Sarcocystis $\cdot$ Rat snake $\cdot$ Mice $\cdot$ Host $\cdot$ Bioassay

\section{Introduction}

Sarcocystis species are cosmopolitan protozoan parasites of homoeothermic and poikilothermic animals (Dubey et al. 2016). Sarcocystis species have a two-host life cycle, with herbivores as intermediate hosts and carnivores as definitive hosts. Clinically, sarcocystosis is typically a mild intestinal illness of humans with occasional incidental findings of sarcocysts on histopathological examination of the muscle tissue. Recently, mysterious and diagnostically challenging outbreaks of human illness associated with muscular sarcocyst infection have been reported in persons that had a history of travel to Malaysian regions; these human infections have been attributed to Sarcocystis species that have snakes as definitive hosts (Abubakar et al. 2013; Arness et al. 1999; Esposito et al. 2012; Italiano et al. 2014; Lau et al. 2014; Yang et al. 2005).

Little is known of the Sarcocystis infection in snakes in the USA. In the present study, we isolated Sarcocystis sporocysts from the intestinal contents of a road-killed rat snake (Pantherophis alleghaniensis) in the USA and investigated the parasite's development in vivo and in vitro. Additionally, we conducted multilocus sequencing and phylogenetic analysis 
to provide genetic information for future investigations in epidemiology and deferential diagnosis.

\section{Materials and methods}

\section{Naturally infected snake}

A deceased adult Eastern rat snake was found on the property of the Beltsville Agricultural Research Center, Maryland, in May 2015. The snake was evaluated of Sarcocystis infection. For this, the intestinal contents were homogenized in a blender, filtered through two-layered gauze, and subjected to sucrose floatation for examination of Sarcocystis sporocysts. After microscopic examination, the floats were mixed with $2 \%$ sulfuric acid, and stored at $4{ }^{\circ} \mathrm{C}$ as described by Dubey et al. (2016).

\section{Attempted transmission to mice}

Sporocysts were bioassayed in mice. For this, Sarcocystis sporocysts in $2 \%$ sulfuric acid were neutralized with $3.3 \%$ $\mathrm{NaOH}$, washed with phosphate buffered saline (PBS), diluted serially, and inoculated orally by feeding needle into outbred Swiss Webster (SW) and interferon gamma gene knockout (KO) mice (Table 1). The recipient mice were observed daily for signs of illness. Because the inoculated KO mice developed neurological signs, we specifically examined these mice for suspected Sarcocystis neurona infection. Small pieces of the brain, and the cerebellum in particular, were squashed on glass slides, mixed with saline, and examined microscopically for merozoites. Muscle squashes were examined microscopically for sarcocysts (Dubey et al. 2016).

After collecting samples for histology (see below) the whole mouse carcass was homogenized in saline $(100 \mathrm{ml})$ in blender and incubated in acid pepsin solution at $37^{\circ} \mathrm{C}$ for $1 \mathrm{~h}$ to release bradyzoites from sarcocysts. After centrifugation, the sediment was suspended in about $10 \mathrm{ml}$ saline $(0.85 \%$ $\mathrm{NaCl})$ and $\sim 25 \mu \mathrm{l}$ of each digest was screened for the presence of bradyzoites at $40 \times$ magnification using a light microscope.

\section{Histological examination}

Portions of the heart, lung, spleen, tongue, eye, brain, kidney, liver, intestine, and muscles were fixed in $10 \%$ buffered neutral formalin. Fixed tissue samples were cut into sections $(2.5 \times 0.7 \mathrm{~cm})$ placed in cassettes, embedded in paraffin, and sectioned $5 \mu \mathrm{m}$ thick. Tissue sections were stained with hematoxylin and eosin and observed using light microscopy.

For immunohistochemical examination, paraffin sections were reacted with anti S. neurona serum prepared from a rabbit injected with culture-derived $S$. neurona merozoites, as described by Dubey et al. (1999). The specificity of the $S$. neurona antiserum has been described by Dubey and Hamir (2000).

Table 1 Bioassay of sporocysts collected from rat snake in interferon gamma gene knockout (KO) mice and Swiss Webster (SW) outbred mice

\begin{tabular}{|c|c|c|c|c|c|c|c|}
\hline \multirow[t]{2}{*}{ Dose } & \multirow[t]{2}{*}{ Mouse } & \multirow[t]{2}{*}{ Necropsy (d.p.i.) } & \multicolumn{2}{|l|}{ Diagnosis } & \multirow[t]{2}{*}{ Clinical signs $^{\mathrm{a}}$} & \multirow[t]{2}{*}{ Sub passage in $\mathrm{KO}$ mouse ${ }^{\mathrm{b}}$} & \multirow[t]{2}{*}{ Cell culture } \\
\hline & & & Brain & Muscle & & & \\
\hline \multirow[t]{2}{*}{$\mathrm{NC}$} & $\mathrm{KO}$ & 39 & Positive & Negative & Yes & \multirow[t]{2}{*}{ ND } & \multirow[t]{2}{*}{ ND } \\
\hline & $\mathrm{KO}$ & 33 & Positive & Negative & Yes & & \\
\hline \multirow[t]{4}{*}{$8 \times 10^{4}$} & KO & 46 & Positive & Negative & Yes & Yes, positive & Yes, positive \\
\hline & $\mathrm{KO}$ & 44 & ND & ND & Yes & \multirow{3}{*}{ ND } & \multirow[t]{3}{*}{ ND } \\
\hline & SW & 155 & Negative & Negative & No & & \\
\hline & SW & 155 & Negative & Negative & No & & \\
\hline \multirow[t]{4}{*}{$8 \times 10^{3}$} & $\mathrm{KO}$ & 36 & Positive & Negative & Yes & Yes, positive & Yes, positive \\
\hline & $\mathrm{KO}$ & 46 & Positive & Negative & Yes & Yes, positive & Yes, positive \\
\hline & SW & 155 & ND & ND & No & \multirow[t]{2}{*}{ ND } & \multirow[t]{2}{*}{ ND } \\
\hline & SW & 155 & ND & ND & No & & \\
\hline \multirow[t]{4}{*}{$8 \times 10^{2}$} & $\mathrm{KO}$ & 52 & Positive & Negative & Yes & ND & Yes, positive \\
\hline & $\mathrm{KO}$ & 46 & Positive & Negative & Yes & Yes, positive & Yes, positive \\
\hline & SW & 155 & Negative & Negative & No & \multirow[t]{2}{*}{ ND } & \multirow[t]{2}{*}{ ND } \\
\hline & SW & 155 & Negative & Negative & No & & \\
\hline \multirow[t]{2}{*}{$8 \times 10^{1}$} & $\mathrm{KO}$ & 155 & Negative & Negative & No & \multirow[t]{2}{*}{ ND } & \multirow[t]{2}{*}{ ND } \\
\hline & SW & 155 & Negative & Negative & No & & \\
\hline
\end{tabular}

$N C$ not counted, $N D$ not done, d.p.i. day post-inoculation

${ }^{\mathrm{a}}$ Weak, neurological signs

${ }^{\mathrm{b}}$ Necropsy was done on 38 or more days post-inoculation 


\section{In vitro culture}

We examined the development of the parasite from $\mathrm{KO}$ mice in African green monkey kidney (CV-1) cells (ATCC CCL-70, Manassas, Virginia, USA). Host cells were grown in $25-\mathrm{cm}^{2}$ cell culture flasks or on 22- $\mathrm{mm}^{2}$ glass coverslips in six-welled cell culture plates in RPMI 1640 cell culture medium (Mediatech, Inc., Manassas, VA, USA) containing 100 IU penicillin/milliliter, $100 \mu \mathrm{g} / \mathrm{ml}$ streptomycin/milliliter and $10 \%(v /$ v) fetal bovine serum (FBS). Cells were maintained in the same medium except the concentration of FBS was $2 \%$.

The infected mouse brain was placed in $3 \mathrm{ml}$ of HBSS and passed through a sterile $10-\mathrm{ml}$ syringe into a $15-\mathrm{ml}$ screw cap centrifuge tube. Sterile 2-mm glass beads were added up to about $0.5 \mathrm{ml}$ in the test tube and the sample vortexed for about $10 \mathrm{~s}$. The samples were examined for the presence of merozoites using light microscopy on the unstained sample. The sample (about $0.5 \mathrm{ml} /$ flask) was inoculated onto each of five monolayers of CV-1 cells in $25 \mathrm{~cm}^{2}$ flasks. The inocula were removed $2 \mathrm{~h}$ later, when $5 \mathrm{ml}$ of maintenance medium was added. Flasks were examined daily for 21 days and then every other day thereafter.

Once merozoites were observed in inoculated cultures, the media was used to infect other flasks of CV-1 cells to keep the cultures growing. This was done at various times to obtain parasites for developmental studies. Parasite development was examined using CV-1 cells grown on sterile $22-\mathrm{mm}^{2}$ glass coverslips. Coverslips containing CV-1 cells each inoculated with merozoites in maintenance medium. The numbers of merozoites was not determined. The inoculum was removed $24 \mathrm{~h}$ post-inoculation (p.i) and replaced with maintenance medium. One or two coverslips were removed at 1-8 days p.i and processed for light microscopic examination after staining with Giemsa. The 22- $\mathrm{mm}^{2}$ coverslips were fixed in $10 \%(v /$ v) buffered formalin solution for $10 \mathrm{~min}$ then post-fixed in $100 \%$ methanol for $10 \mathrm{~min}$, air dried, stained with Giemsa for $1 \mathrm{~h}$, and mounted on glass slides using Permount ${ }^{\mathrm{TM}}$ (Fisher Scientific Company, Fair Lawn, NJ, USA) for microscopic examination.

\section{DNA extractions and PCR amplification}

The merozoites/schizonts derived from the brain of infected $\mathrm{KO}$ mice and in vitro cultures were used for molecular characterization. Genomic DNA was extracted using the DNeasy ${ }^{\circledR}$ Blood and Tissue Kit (Qiagen, Valencia, CA, USA) according manufacturer's instructions. DNA quantity and quality were determined by the NanoDrop Lite Spectrophotometer (Thermo Fisher Scientific, Wilmington, DE, USA). Molecular characterization was done by amplification and sequencing of three regions of the nuclear ribosomal DNA unit: $18 S \mathrm{rRNA}$, internal transcribed spacer-1 (ITS-1), and 28S rRNA, and a mitochondrial cytochrome c oxidase subunit 1 (coxl) locus following the procedures as described previously by Gjerde and Josefsen (2015). The PCR amplifications were performed in $50 \mu \mathrm{l}$ total reaction volume containing 10 pmol of each primer pair and $1 \times$ Taq PCR Master Mix Kit (Qiagen, Inc., Valencia, USA). The thermal cycler (Veriti ${ }^{\circledR}$ Thermal Cycler, Applied Biosystems, Foster City, USA) conditions were set at initial denaturation at $95^{\circ} \mathrm{C}$ for $10 \mathrm{~min}, 40$ cycles of amplification $\left(95^{\circ} \mathrm{C}\right.$ for $45 \mathrm{~s}$, $52-56{ }^{\circ} \mathrm{C}$ for $45 \mathrm{~s}$, and $72{ }^{\circ} \mathrm{C}$ for $1 \mathrm{~min}$ ) and final extension at $72{ }^{\circ} \mathrm{C}$ for $10 \mathrm{~min}$. Appropriate positive (DNA of S. neurona merozoites) and negative $\left(\mathrm{H}_{2} \mathrm{O}\right)$ controls were included in all analyses. The amplified PCR products were run on $2.5 \%(w / v)$ agarose gel with ethidium bromide staining, and visualized by using the Gel Logic 212 Imaging Systems (Eastman Kodak Company, Rochester, NY, USA).

\section{DNA sequencing and phylogenetic analysis}

The PCR amplicons of 18S rRNA, 28S rRNA, ITS-1, and cox 1 were excised from the gel, and purified using the QIAquick Gel Extraction (Qiagen, Inc., Valencia, USA) according to the manufacturer's recommendation. The purified PCR products were sent to Macrogen Corporation (Rockville, MD, USA) for direct sequencing using the same primer pair used in amplification to obtain both reads. The resulting sequences were imported, read, edited manually if necessary, and analyzed using the software Geneious version 9.0.4 (Biomatters Ltd. Auckland, NZ). New sequences were compared with other sequences deposited in NCBI GenBank by BLASTn analysis to detect intraspecies and interspecies variation on these DNA regions.

A phylogenetic tree based on $18 S r R N A$ sequences was constructed using sequences obtained from the rat snake Sarcocystis sp. and sequences of various species of Sarcocystis deposited in the NCBI GenBank. One hundred bootstrap replicates of the phylogenetic relationships among these sequences were reconstructed under the criterion of maximum likelihood using PhyML as implemented in the Geneious version 9.0.4 (Guindon et al. 2010). The HKY85 model of nucleotide substitution was used, estimating the transition/transversion ratio and the gamma distribution parameter to model rate variation among sites. No sites were presumed to be invariant. The complete deletion method was used for ambiguous/missing data, and topologies were searched using the nearest neighbor interchange method. The final dataset incorporated 54 sequences corresponding to region of 1357 nucleotides, and Besnoitia bennetti (AY665399) as an out group.

\section{Results}

Numerous sporocysts were found in the intestinal contents of the rat snake. Fully sporulated oocysts/sporocysts were seen in fresh samples before being placed into sulfuric acid. The oocysts measured approximately $16 \times 10.5 \mu \mathrm{m}(n=20)$ and 
sporocysts $18 \times 11 \mu \mathrm{m}(n=20)$. Oocysts/sporocysts were infective to KO mice but not to SW mice (Table 1). Depending on the dose, the inoculated $\mathrm{KO}$ mice developed neurological signs resulting in death or the need for euthanasia when they became moribund between 33 and 52 days p.i. Sarcocystis schizonts and merozoites were found in the brains, particularly the cerebellum (Fig. 1). Sub inoculation of brain homogenate of infected $\mathrm{KO}$ mice to naive $\mathrm{KO}$ mice was found to produce infections (Table 1).

Histological examination revealed meningoencephalitis, characterized by infiltration of mononuclear cells around the blood vessels, focal necrosis of the neuropil, particularly in the cerebellum. Immature and mature schizonts, free and intracellular merozoites were scattered in the brain. Isolated inflammatory foci contained degenerating and intact merozoites among neutrophils. The schizonts were up to $45 \mu \mathrm{m}$ in size and contained up to 34 merozoites. The merozoites were up to $12 \mu \mathrm{m}$ long and had a terminal nucleus (Fig. 1A-D). Neither sarcocysts nor bradyzoites were detected by any of the methods employed. The organisms in KO mice did not react with $S$. neurona antibody. Three of the five cultures were positive for merozoites at 6 days p.i. The merozoites in the inocula were infectious for other CV-1 cell cultures (Table 1). Parasite density was low and most stages were schizonts in various stages of development. Developing schizonts varied in size, varying with the maturity of the nucleus. They increased in size as the nucleus developed numerous nucleoli and became lobed. The largest multilobed schizont observed was $54 \times 26 \mu \mathrm{m}$. Mature schizonts could contain over 50 merozoites (Fig. 1E, F). Merozoites were approximately $7.2 \times 1.9 \mu \mathrm{m}(n=20)$ and were produced on blastophores that developed on the surface of schizonts.

PCR analysis with schizont/merozoite DNA as the template yielded amplicons of the expected size for the $18 \mathrm{~S}$ $r R N A$ (in three fragments), $28 S$ rRNA (in two fragments), ITS-1, and cox1 loci. PCR-DNA sequencing of amplicons resulted in the unambiguous sequences of three nuclear DNA regions: $18 S$ rRNA (1790 bp), $28 S$ rRNA (1530 bp), and ITS-1 (900 bp), and a mitochondrial DNA locus, coxl (1015 bp). These sequences were submitted to the NCBI GenBank with accession numbers KU891600 (18S rRNA), KU891601 (28S rRNA), KU891602 (ITS-1), and KU891603 (coxl) and designated as originating from Sarcocystis sp., ex., Pantherophis alleghaniensis.

Analysis of $18 S$ rRNA sequence of Sarcocystis sp., ex., P. alleghaniensis, confirmed its membership among species of Sarcocystis and indicated an especially close relationship to other parasites in this genus that employ snakes as their definitive hosts and also to other Sarcocystis which use rodents as intermediate hosts but for which the definitive hosts are yet unknown (Fig. 2). The $18 S$ rRNA sequence of Sarcocystis sp., ex., $P$. alleghaniensis, shared $98 \%$ identity with sequences of Sarcocystis sp. 1 JJH clone 1 (KF309698) and $97 \%$ with
Sarcocystis sp. 1 JJH-2013 clone 2 (KF309699) followed by $94 \%$ identity with other sequences such as Sarcocystis nesbitti (HF544323), Sarcocystis sp., ex., Morelia viridis GM (KC201639), Sarcocystis singaporensis clone GN8-5 (AF434054), and many other species of Sarcocystis.

In phylogenetic tree based on $18 S$ rRNA sequences, Sarcocystis sp., ex., P. alleghaniensis, clustered consistently with Sarcocystis eothenomysi (from a vole in China, and suspected to have a snake definitive host), and with less bootstrap support to $S$. nesbitti; these three were most typically grouped with the following other species: Sarcocystis atheridis, S. singaporensis, Sarcocystis sp., ex., Morelia viridis GM2013, Sarcocystis cf. clethrionomyelaphis JJH-2013, Sarcocystis zuoi, and Sarcocystis sp. MA\#347 (Fig. 2); all these employ snakes (python, cobra, rat snake, viper) as their definitive hosts (Lau et al. 2013; More et al. 2014; Hu et al. 2014, 2015; Dubey et al. 2016). Relationship of Sarcocystis sp., ex., $P$. alleghaniensis, to the $S$. eothenomysi, and $S$. nesbitti was stable when substituting various out group taxa (B. bennetti, Toxoplasma gondii, Eimeria anguillae). The partial $28 S$ rRNA sequence of Sarcocystis sp., ex., P. alleghaniensis, shared the highest identity (94\%) with sequence of $S$. singaporensis (AF237617), and $93 \%$ identity with Sarcocystis zamani (AF237616) followed by $\leq 92 \%$ identity with other sequences of Sarcocystis spp. The new ITS-1 sequence of Sarcocystis sp., ex., P. alleghaniensis, shared poor nucleotide sequence similarities with other Sarcocystis species, but no ITS-1 sequences have yet been reported from any Sarcocystis species that use snakes as definitive hosts. The partial coxl sequence of Sarcocystis sp., ex., P. alleghaniensis, shared the highest identity (98-97\%) with Sarcocystis speeri (KT207461), Sarcocystis lutrae (KM657808, KF601326), Sarcocystis arctica (KF601318-KF601321), Sarcocystis rileyi (KJ396582), and S. neurona strain BR2012 (KF854272). The uses of coxl gene as a genetic marker for Sarcocystis species discrimination has been proposed only recently, and so sequences of limited species are available for comparative use (Gjerde 2013).

\section{Discussion}

In the present study, natural infections of Sarcocystis were detected in the intestines of Eastern rat snake (P. alleghaniensis) for the first time from the USA. The Eastern rat snake is an endangered, nonvenomous colubrid species, endemic to North America. It occupies a diverse habit including farmlands, hardwood forests, forested wetlands, thickets and fields adjacent to forests, isolated urban woodlots, and backyards. They prey on rodents, lizards, frogs, birds, bird's eggs, young chickens, and chicks (Conant and Collins 1998). We noted the presence of 3-4 partial digested house 

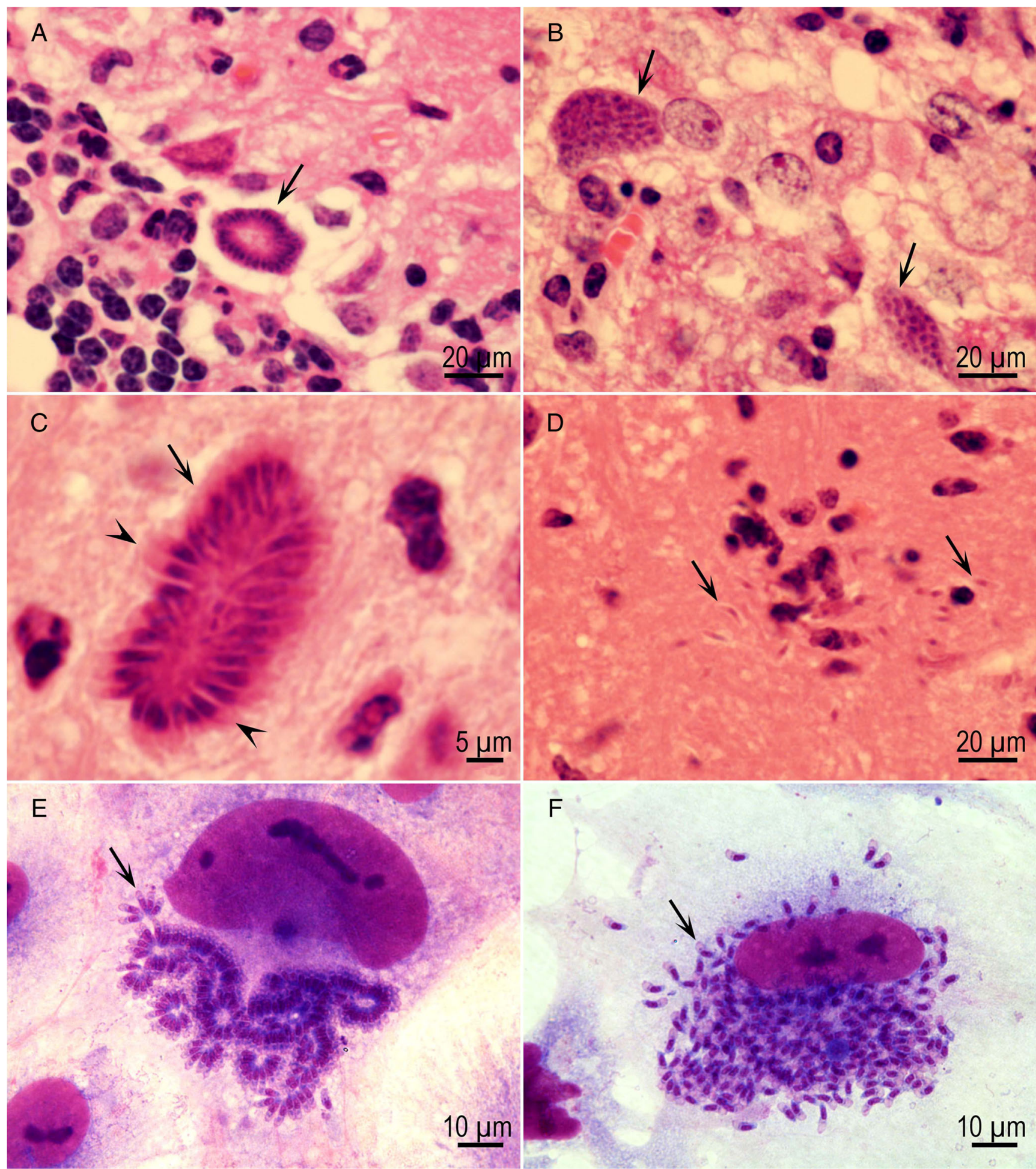

F

Fig. 1 Sarcocystis schizonts and merozoites. A-D Histological sections of the brain of KO mice inoculated with Sarcocystis sporocysts. Hematoxylin and eosin stain. E, F Stages in CV-1 cells, Giemsa stain. A Schizont (arrow) at the periphery of mononuclear cell infiltrations around a blood vessel. B Two immature schizonts (arrows). C Mature

mice in the gut of the snake at the time of necropsy. However, we were unable to transmit the infection to SW mice.
The development of schizonts in the brain and of neurological signs in the inoculated $\mathrm{KO}$ mice suggested a S. neurona- 


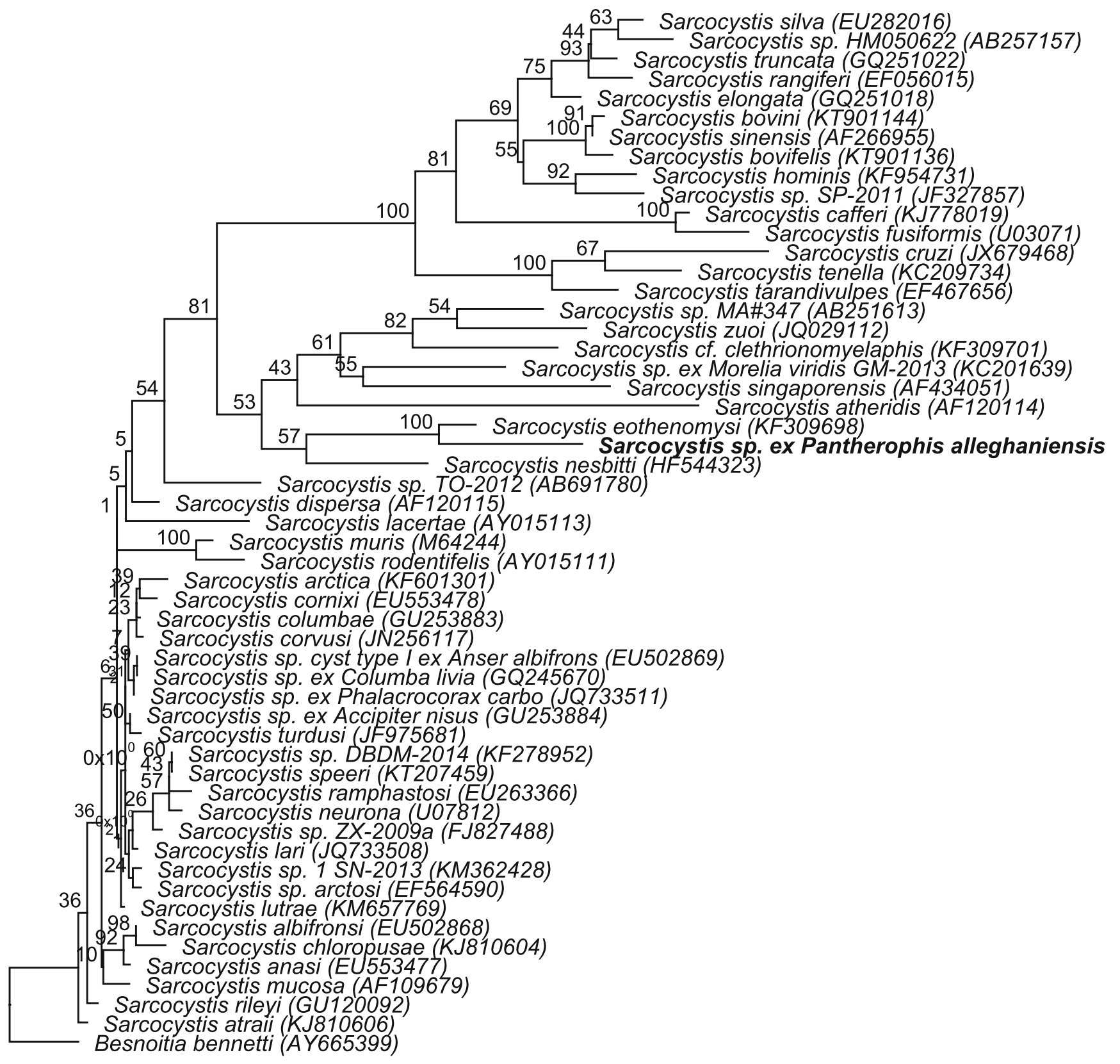

\section{1}

Fig. 2 Phylogenetic tree based on $18 S$ r $R N A$ sequences. Input sequences were the $18 S$ rRNA regions of various species of Sarcocystis retrieved from NCBI GenBank, and new 18S rRNA sequence obtained from Sarcocystis sp. from rat snake (Sarcocystis sp., ex., Pantherophis alleghaniensis). Accession numbers of sequences were given in parenthesis following the species name. One hundred bootstrap replicates of the phylogenetic relationships among these sequences were

like infection, but the parasite did not react to $S$. neurona antibodies and was genetically distinct from $S$. neurona. In the absence of sarcocysts, it was not possible to determine the Sarcocystis species or better classify the parasite. Molecular characterization of the parasite from the KO mice revealed a closer relationship with Sarcocystis species that use snakes as reconstructed under the criterion of maximum likelihood using PhyML as implemented in the Geneious version 9.0.4. Sarcocystis sp., ex., Pantherophis alleghaniensis, showed close relationship with those employ snakes (python, cobra, rat snake, and viper) as their definitive hosts, and rodents as intermediate hosts or which the natural hosts are yet unknown

definitive hosts (Dubey et al. 2016). ITS-1 sequences are a more variable portion of ribosomal DNA, and show higher intraspecies and interspecies divergence in genus Sarcocystis. We were unable to perform phylogenetic analyses based on hyper-variable ITS-1 sequences due to lack of ITS-1 sequences of snake-derived Sarcocystis species in database. 
However, the $18 S$ rRNA provided a good basis to compare the current isolates to previously sampled species of Sarcocystis. The $18 S$ rRNA sequences have been used frequently to show phylogenetic relationship and diagnosis of Sarcocystis species (Dubey et al. 2016).

Sarcocystis atheridis sporocysts were isolated from a Nitsche's bush viper (Atheris nitschei nitschei), from Uganda, and experimental transmission resulted in sarcocyst development in the skeletal muscles of laboratory mice (CD1 and Barbary striped mice) not in rats (Wistar H) (Dolezel et al. 1999; Slapeta et al. 1999). The sequences of Sarcocystis sp. 1 JJH-2013 clone 1 and clone 2 were submitted from Sarcocystis eothenomysi infecting the large oriental vole (Eothenomys miletus) from Anning, China, and suspected to have a snake definitive host (Hu et al. 2014). Sarcocystis sp. MA347 was reported in raccoons (intermediate hosts) introduced to Japan from North America (Jinnai et al. 2009). Definitive hosts are unknown, possibly carnivorous snakes by phylogenetic analysis. Sarcocystis zuoi was described from wildcaught Norway rats (Rattus norvegicus) in China, and king rat snakes (Elaphe carinata) were experimentally defined as definitive host (Hu et al. 2005, 2012). Sarcocystis singaporensis is a well described species that uses python snakes as definitive hosts and rodents as intermediate hosts (Dubey et al. 2016). Recently, Sarcocystis sp., ex., Morelia viridis, was described from Sarcocystis spp. sporocysts from pooled feces from four green pythons (Morelia viridis) from Germany (More et al. 2014). Review of the literature and results of the present study point to a high diversity of undefined Sarcocystis species infecting to snakes.

In conclusion, the present study described the natural infection of Sarcocystis species in rat snake. We believe the parasites we observed have not previously been reported from rat snake, and provisionally refer to them as Sarcocystis sp., ex., Pantherophis alleghaniensis. Molecular and parasitological characteristics suggest the parasite cycle between snakes as definitive hosts and possibly rodents as intermediate hosts. These data underscore the likelihood that parasites in this genus that employ snakes as their definitive hosts constitute an ancient, globally distributed monophyletic group. These data also raise the possibility that neurological sequalae may be more common in intermediate hosts of Sarcocistis spp. than has previously been appreciated.

Compliance with ethical standards All investigations reported here were approved by the institutional animal care and use protocol committee of the US Department of Agriculture.

\section{References}

Abubakar S, Teoh BT, Sam SS, Chang LY, Johari J, Hooi PS, LakhbeerSingh HK, Italiano CM, Syed Omar SF, Wong KT, Ramli N, Tan CT
(2013) Outbreak of human infection with Sarcocystis nesbitti, Malaysia, 2012. Emerg Infect Dis 19:1989-1991

Arness MK, Brown JD, Dubey JP, Neafie RC, Granstrom DE (1999) An outbreak of acute eosinophilic myositis attributed to human Sarcocystis parasitism. Am J Trop Med Hyg 61:548-553

Conant R, Collins (1998) A field guide to reptile and amphibians: eastern north america. Houghton Mifflin Company, Boston

Dolezel D, Koudela B, Jirku M, Hypsa V, Obornik M, Votypka J, Modry D, Slapeta JR, Lukes J (1999) Phylogenetic analysis of Sarcocystis spp. of mammals and reptiles supports the coevolution of Sarcocystis spp. with their final hosts. Int J Parasitol 29:795-798

Dubey JP, Hamir AN (2000) Immunohistochemical confirmation of Sarcocystis neurona infections in raccoons, mink, cat, skunk and pony. J Parasitol 86:1150-1152

Dubey JP, Mattson DE, Speer CA, Baker RJ, Mulrooney DM, Tornquist SJ, Hamir AN, Gerros TC (1999) Characterization of Sarcocystis neurona isolate (SN6) from a naturally infected horse from Oregon. J Eukaryot Microbiol 46:500-506

Dubey JP, Calero-Bernal R, Rosenthal BM, Speer CA, Fayer R (2016) Sarcocystosis of Animals and Humans, 2nd edn. CRC Press, Boca Raton

Esposito DH, Freedman DO, Neumayr A, Parola P (2012) Ongoing outbreak of an acute muscular Sarocystis-like illness among travellers returning from Tioman Island, Malaysia, 2011-2012. Euro Surveill 17:

Gjerde B (2013) Phylogenetic relationships among Sarcocystis species in cervids, cattle and sheep inferred from the mitochondrial cytochrome c oxidase subunit I gene. Int J Parasitol 43:579-591

Gjerde B, Josefsen TD (2015) Molecular characterisation of Sarcocystis lutrae $\mathrm{n}$. sp. and Toxoplasma gondii from the musculature of two Eurasian otters (Lutra lutra) in Norway. Parasitol Res 114:873-886

Guindon S, Dufayard JF, Lefort V, Anisimova M, Hordijk W, Gascuel O (2010) New algorithms and methods to estimate maximumlikelihood phylogenies: assessing the performance of PhyML 3.0. Syst Biol 59:307-321

Hu JJ, Ma TC, Li XR (2005) A new species of sarcocysts (Sporozoea, Eucoccidiida) from Rattus norvegicus. Acta Zootaxon Sin 30:287-290

Hu JJ, Meng Y, Guo YM, Liao JY, Song JL (2012) Completion of the life cycle of Sarcocystis zuoi, a parasite from the Norway rat, Rattus norvegicus. J Parasitol 98:550-553

Hu JJ, Liu Q, Yang YF, Esch GW, Guo YM, Zou FC (2014) Sarcocystis eothenomysi $\mathrm{n}$. sp. (Apicomplexa: Sarcocystidae) from the large oriental vole Eothenomys miletus (Thomas) (Cricetidae: Microtinae) from Anning, China. Syst Parasitol 89:73-81

Hu JJ, Liu TT, Liu Q, Esch GW, Chen JQ (2015) Sarcocystis clethrionomyelaphis Matuschka, 1986 (Apicomplexa: Sarcocystidae) infecting the large oriental vole Eothenomys miletus (Thomas) (Cricetidae: Microtinae) and its phylogenetic relationships with other species of Sarcocystis Lankester, 1882. Syst Parasitol 91:273-279

Italiano CM, Wong KT, Abubakar S, Lau YL, Ramli N, Syed Omar SF, Kahar BM, Tan CT (2014) Sarcocystis nesbitti causes acute, relapsing febrile myositis with a high attack rate: description of a large outbreak of muscular sarcocystosis in Pangkor Island, Malaysia, 2012. PLoS Negl Trop Dis 8:e2876

Jinnai M, Kawabuchi-Kurata T, Tsuji M, Nakajima M, Fujisawa K, Nagata S, Koide H, Matoba Y, Asakawa M, Takahashi K, Ishihara C (2009) Molecular evidence for the presence of new Babesia species in feral raccoons (Procyon lotor) in Hokkaido, Japan. Vet Parasitol 162:241-247

Lau YL, Chang PY, Subramaniam V, Ng YH, Mahmud R, Ahmad AF, Fong MY (2013) Genetic assemblage of Sarcocystis spp. in Malaysian snakes. Parasit Vectors 6:257

Lau YL, Chang PY, Tan CT, Fong MY, Mahmud R, Wong KT (2014) Sarcocystis nesbitti infection in human skeletal muscle: possible transmission from snakes. Am J Trop Med Hyg 90:361-364

More G, Pantchev N, Herrmann DC, Vrhovec MG, Ofner S, Conraths FJ, Schares G (2014) Molecular identification of Sarcocystis spp. 
helped to define the origin of green pythons (Morelia viridis) confiscated in Germany. Parasitology 141:646-651

Slapeta JR, Modry D, Koudela B (1999) Sarcocystis atheridis sp. nov., a new sarcosporidian coccidium from Nitsche's bush viper, Atheris nitschei Tornier, 1902, from Uganda. Parasitol Res 85:758-764
Yang ZQ, Wei CG, Zen JS, Song JL, Zuo YX, He YS, Zhang HF, Attwood SW, Chen XW, Yang GC, Zhou X, Quan X, Li CY, Han D, Liu AW, Lin P (2005) A taxonomic re-appraisal of Sarcocystis nesbitti (Protozoa: Sarcocystidae) from the monkey Macaca fascicularis in Yunnan, PR China. Parasitol Int 54:75-81 